\title{
Editorial: Tailored Porous Biomaterials for Hard and Soft Tissues: Focus on Surface Functionalization
}

\author{
Yadir Torres ${ }^{1}$, Ana M. Beltrán ${ }^{1 *}$ and Juan V. Cauich-Rodriguez ${ }^{2}$ \\ ${ }^{1}$ Departamento de Ingeniería y Ciencia de los Materiales y del Transporte, Escuela Politécnica Superior, Universidad de Sevilla, \\ Seville, Spain, ${ }^{2}$ Unidad de Materiales, Centro de Investigación Científica de Yucatán, A.C. (CICY), Mérida, Mexico
}

Keywords: porous biomaterials, surface modification, biomechanical behaviour, osseointegration, bacterial and biological response, hard and soft tissues replacement

Editorial on the Research Topic

Tailored Porous Biomaterials for Hard and Soft Tissues: Focus on Surface Functionalization

Nowadays, replacement of soft and hard tissues is frequently required due to trauma, diseases, congenital defects, cancer, etc. to improve quality of life of human beings. In the case of these types of implants for orthopaedic and dental applications may fail due to different reasons such as bone resorption associated with stress-shielding phenomena, deficient osseointegration because of interfacial problems and/or inappropriate design. How to overcome these limitations is challenging and different solutions are reported by researchers.

Porous biomaterials are extensively used for a huge variety of biomedical applications as they can exhibit similar mechanical properties to the tissues to be replaced and/or the provision of spaces for tissue ingrowth, exchange of nutriments and waste products, cell compartmentalization and development of complex structures. In this regard, the use of natural of synthetic polymers for the development of biomaterials or coatings is increasing, such as elastin-like polypeptides and their recombinant thanks to the possibility to be controlled at molecular level. Besides, the combination of them allows designing the suitable structure and properties to support the attachment and activities of cell types (Mbundi et al.). On the other hand, the use of porous nano-scaffolds seems to facilitate tissue regeneration. In their review, Ghosh and Webster discuss the advantages of using various nanohybrid composed of mesoporous silica nanoparticles for tissue engineering and their application in diverse types of scaffolds and implants, although few issues should still need addressed.

The use of fibrous scaffolds based on biodegradable and biocompatible materials is also a potential solution since those fabricated by electronspun allow the anchorage, differentiation and proliferation of RIN-M beta cells (Cortés-Ortiz et al.).

Furthermore, the proliferation of bacteria on implants needs also to be considered since it is also one of the reasons for tissue replacement failure. Therefore, the possibility of using scaffolds with antibacterial properties is a great advantage. Arango-Ospina et al. suggest the combination of Manuka honey and bioactive glass as an interesting approach to create multifunctional bone tissue engineering scaffolds.

Tissue replacement can also be related to a particular cause such a diabetes. In such cases, effective bone tissue therapies are needed. Thus, Li et al. reported the combination of peptides with a scaffold to promote osteogeneis and angiogenesis for improving the bone regeneration in this particular cases of fracture.

From these results, it is evident that the development of a porous structure in biomaterials is a desirable

Front. Mater. 8:752166. doi: 10.3389/fmats.2021.752166 property. When the resulting porous structure is further surface functionalized a better performance is 
achieved not only for soft tissue replacement but also for bone and teeth substitutes. The proper balance between macro, micro, and nanoporosity will render not only mechanical matching with the native tissue to be replaced but also a suitable milieu for cell adhesion and proliferation. Further improvements need to be pursued to obtain hierarchical materials where one type of functionalization will facilitate cell attachment while other functionalization will provide antibacterial properties while simultaneously releasing bioactive compounds.

\section{AUTHOR CONTRIBUTIONS}

All authors listed have made a substantial, direct, and intellectual contribution to the work and approved it for publication.
Conflict of Interest: The authors declare that the research was conducted in the absence of any commercial or financial relationships that could be construed as a potential conflict of interest.

Publisher's Note: All claims expressed in this article are solely those of the authors and do not necessarily represent those of their affiliated organizations, or those of the publisher, the editors and the reviewers. Any product that may be evaluated in this article, or claim that may be made by its manufacturer, is not guaranteed or endorsed by the publisher.

Copyright (C) 2021 Torres, Beltrán and Cauich-Rodriguez. This is an open-access article distributed under the terms of the Creative Commons Attribution License (CC $B Y)$. The use, distribution or reproduction in other forums is permitted, provided the original author(s) and the copyright owner(s) are credited and that the original publication in this journal is cited, in accordance with accepted academic practice. No use, distribution or reproduction is permitted which does not comply with these terms. 